\title{
Optical Frequency Metrology of an Iodine-Stabilized He-Ne Laser Using the Frequency Comb of a Quantum-Interference-Stabilized Mode-Locked Laser
}

Volume 112

\section{Ryan P. Smith}

JILA, University of Colorado and National Institute of Standards and Technology,

Boulder, CO 80309-0440

and

Department of Physics,

University of Colorado,

Boulder, CO 80309-0390

Peter A. Roos, Jared K.

Wahlstrand, Jessica A. Pipis

JILA, University of Colorado and

National Institute of Standards

and Technology,

Boulder, CO 80309-0440

\section{Maria Belmonte Rivas}

Department of Aerospace

Engineering,

University of Colorado, Boulder, CO 80309-0429

Number 6

November-December 2007

and

\section{Steven T. Cundiff}

JILA, University of Colorado and National Institute of Standards and Technology,

Boulder, CO 80309-0440

Ryan.P.Smith@colorado.edu

roos@spectrum.montana.edu

Jared.Wahlstrand@colorado.edu

Jessica.Pipis@colorado.edu

belmonte@colorado.edu

steven.cundiff@nist.gov demonstrate carrier-envelope offset frequency fluctuations of less than $5 \mathrm{mHz}$ using a $1 \mathrm{~s}$ gate time. With the resulting stable frequency comb, we measure the optical frequency of the iodine transition [ ${ }^{127} \mathrm{I}_{2} \mathrm{R}(127)$ 11-5 i component] to be $473612214712.96 \pm 0.66 \mathrm{kHz}$, well within the uncertainty of the CIPM recommended value. The stability of the quantum interference technique is high enough such that it does not limit the measurements.

Key words: femtosecond (fs) lasers; laser frequency control; optical clocks; optical frequency comb; optical frequency measurement; stabilized lasers.

We perform optical frequency metrology of an iodine-stabilized He-Ne laser using a mode-locked Ti:sapphire laser frequency comb that is stabilized using quantum interference of photocurrents in a semiconductor. Using this technique, we
Accepted: December 6, 2007

Available online: http://www.nist.gov/jres

\section{Introduction}

Absolute optical frequency metrology is the measurement of an optical frequency referenced to the primary frequency standard, a cesium atomic clock [1]. This microwave frequency standard differs from optical frequencies by more than five orders of magnitude, making direct comparison impossible. In the past, this problem has been overcome by means of arduous frequency chains [2]. More recently, the introduction of the frequency comb produced by femtosecond mode- locked lasers [3-6] has dramatically simplified the link between optical and microwave frequencies, and frequency combs have become the predominant tool used for optical frequency metrology. The frequency comb is a necessary technique for use in optical atomic clocks. Optical frequency standards now surpass cesium reference standards in stability and rival or potentially surpass them in accuracy $[2,7,8]$.

A mode-locked laser produces a wide comb of evenly spaced optical frequency modes in the frequency domain [9]. The frequency comb may be seen as a 
direct result of a sequence of coherent short pulses in the time domain. The optical frequency of a given comb line is $v_{n}=n f_{\text {rep }}+f_{0}$, where $n$ is an integer comb line index, $f_{\text {rep }}$ is the repetition rate (spacing between comb modes), and $f_{0}$ is the carrier-envelope offset frequency, the rate at which the carrier-envelope phase slips from pulse to pulse due to the difference in the group and phase velocities within the laser cavity. When these two RF frequencies are referenced to the primary cesium standard, the absolute optical frequencies of all the comb lines are known. To precisely determine the unknown frequency of a continuous-wave (CW) laser, one must first measure the frequency (by a standard method such as a wavemeter) to within the repetition rate of the laser. Then one can simply measure the heterodyne beat $f_{\text {beat }}$ between the CW laser and the closest line in the frequency comb. This RF beat results from the interference of these two optical frequencies. The unknown frequency of the laser is then

$$
v_{\text {unknown }}=n \times f_{\text {rep }}+f_{0} \pm f_{\text {beat }} .
$$

Stabilizing the frequency comb is helpful for simplifying optical metrology measurements. Stabilization is accomplished by comparing the two parameters of the comb, $f_{\text {rep }}$ and $f_{0}$, to a stable reference. Typically, stabilization of $f_{\text {rep }}$ is straightforward, performed by detecting the rate of pulses onto a photodetector, and then feeding back to an actuator that controls the cavity length of the mode-locked laser. However, detection and stabilization of $f_{0}$ is more involved, and several techniques have been developed for this purpose. In one common technique to measure the carrier-envelope offset frequency, known as $v$ - to - $2 v$ self-referencing, a frequency-doubled comb line from the low-frequency wing of the spectrum of the mode-locked laser, after having been broadened to span an octave, is optically interfered with a comb line that is nearly twice its frequency in the upper wing of the spectrum. The heterodyne beat frequency between these two lines is $f_{0}$ [3]. In this work, we demonstrate the applicability to optical frequency metrology of a recently developed alternative self-referencing technique that uses quantum interference control of photocurrents in semiconductors [10-14]. This allows the two-color interferometer of the standard technique to be replaced by a semiconductor device.

Quantum interference control (QIC) of injected photocurrents in semiconductors exploits phase-sensitive interference between one- and two-photon absorption pathways from the valence to the conduction band
$[10,11]$. Two-photon absorption of light at frequency $v$ (below the band gap) and one-photon absorption of $2 v$ (above gap) light separately produce carrier populations that are symmetric in $k$-space, resulting in no net current flow. However, when both absorption pathways exist simultaneously, quantum interference between them can occur, creating an asymmetry in $k$-space for the carrier population and therefore a net current in the semiconductor. The current injected in the semiconductor due to this interference depends sinusoidally on the phase difference of the two absorption pathways, $2 \phi_{v}-\phi_{2 v}$, where $\phi_{v}$ and $\phi_{2 v}$ are the optical phases of the two spectral components involved. Light pulses containing optical frequencies that differ by a factor of two (an octave-spanning spectrum) can excite both one- and two-photon absorption simultaneously. The carrierenvelope phase is, up to a constant offset, proportional to $2 \phi_{v}-\phi_{2 v}$, and thus the injected current due to quantum interference oscillates at $f_{0}$ [11]. Thus, using QIC we are able to replace the doubling crystal and optical interferometer of the standard self-referencing apparatus with two-photon absorption and quantum interference in a semiconductor. The resulting measurement has a high enough signal-to-noise ratio to be used to stabilize $f_{0}$ [14], and here we apply this technique of measuring and stabilizing $f_{0}$ to optical frequency metrology.

\section{Experiment}

To demonstrate optical frequency metrology we chose to measure the frequency of an iodine-stabilized He-Ne laser. The absolute frequency of the He-Ne laser is determined by simultaneously measuring the three frequency parameters on the right side of Eq. (1). In addition, the index $n$ of the comb line that is used in the heterodyne detection with the He-Ne laser and the sign of the $f_{\text {beat }}$ are determined by prior knowledge of the HeNe laser frequency. The experimental setup is shown in Fig. 1. The laser being measured is a commercial iodine-stabilized He-Ne laser, ${ }^{1}$ which uses a saturation spectroscopy technique to lock the lasing frequency to a specific hyperfine component of molecular iodine vapor in a temperature-stabilized cell. The He-Ne laser is locked to the ${ }^{127} \mathrm{I}_{2} \mathrm{R}$ (127) $11-5 \mathrm{i}$ transition. A rough measurement of the He-Ne laser frequency using a commercial wavemeter provides enough information to determine the closest comb line index. The vapor

\footnotetext{
${ }^{1}$ Winters Electro-Optics Model 100 Iodine-stabilized He-Ne laser; mention of a brand name is for technical information only and does not represent an endorsement by NIST.
} 


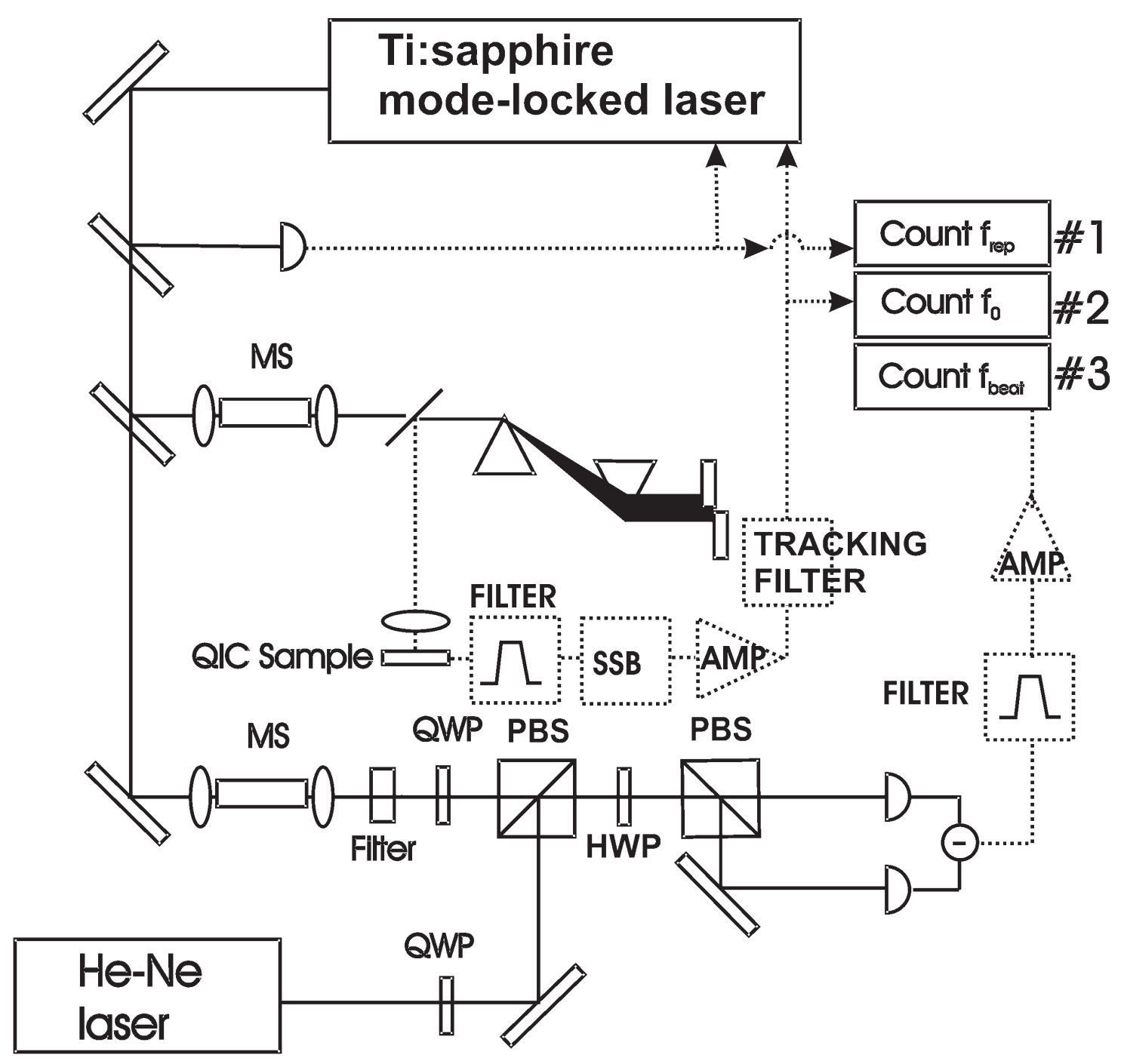

Fig. 1. Experimental setup for optical metrology using quantum interference control (QIC). The QIC setup stabilizes $f_{0}$ and also provides a countable signal. MS, microstructure fiber; QWP, quarter wave plate; HWP, half wave plate; PBS, polarizing beam splitting cube; AMP, RF Amplifier; TF, Tracking Filter; FILTER, bandpass filter; SSB, single side-band mixer.

iodine cell of the He-Ne laser is frequency calibrated by the Bureau International des Poids et Mesures (BIPM) within $12 \mathrm{kHz}$ [15].

As mentioned above, stabilization of the comb is required for use in optical frequency metrology. We phase lock $f_{\text {rep }}$ of a mode-locked Ti:sapphire laser to a synthesized signal near $94 \mathrm{MHz}$ by controlling the cavity length of the laser with a piezoelectric transducer. The pulse train is directly measured with a photodiode, and $f_{\text {rep }}$ is counted (counter 1 in Fig. 1). The synthesizer and all counters are referenced to a commercial Cs atomic clock. ${ }^{2}$ A portion of the output of the

\footnotetext{
${ }^{2}$ HP 5071A Primary frequency standard; mention of a brand name is for technical information only and does not represent an endorsement by NIST.
}

laser is spectrally broadened in a microstructure fiber and then sent through a prism pair, which compensates for delays between the spectral components of $v$ and $2 v$ due to dispersion in the fiber. This light is directed onto the QIC semiconductor device, which is lowtemperature grown GaAs with AuGe electrodes, separated by about $30 \mu \mathrm{m}$, deposited on the surface [13]. The carrier-envelope offset frequency $\left(f_{0}\right)$ signal, derived from the QIC current injected across the electrodes and then amplified using a transimpedence amplifier, is typically detected near $400 \mathrm{kHz}$. It is necessary to detect $f_{0}$ at such a low frequency because the intrinsic capacitance of the sample structure acts as a low-pass filter [16]. The signal is then shifted to near $82 \mathrm{MHz}$ (using a second synthesizer phase locked 
to the first) using a single-sideband mixer [12] in order to match the design frequency of the stabilization electronics. An RF tracking filter is used to improve the $f_{0}$ signal-to-noise ratio (originally $\sim 20 \mathrm{~dB}$ with a $10 \mathrm{kHz}$ resolution bandwidth) in order to make the measurement of this parameter more robust. The tracking filter phase locks a voltage controlled oscillator (VCO) to the up-shifted $f_{0}$. The VCO output from the tracking filter is then mixed with a synthesized signal at $7 / 8 f_{\text {rep }}$ in order to generate an error signal for phase locking of $f_{0}$ by tilting the cavity end mirror [17]. We count the output from the tracking filter (counter 2 in Fig. 1) as part of the unknown optical frequency determination via Eq. (1). Although introducing the tracking filter had the potential to add phase noise to the measurement, we find that the $f_{0}$ noise contributions are insignificant (five orders of magnitude smaller) compared to other sources of noise in the final determination of the unknown optical frequency. In addition, previous $f_{0}$ phase noise measurements using the tracking filter show comparable noise performance to those without a tracking filter (data not shown). For comparison, we also made measurements using the standard $v$ - to $-2 v$ self-referencing technique.

A heterodyne beat between the He-Ne laser and the frequency comb provides the final parameter necessary in Eq. (1) for determining the He-Ne frequency. Light from the mode-locked laser, spectrally broadened using a microstructure fiber, is combined with the output of the He-Ne laser using a pair of polarizing beam splitters. We detect the heterodyne beat between the He-Ne and the nearest comb line $\left[f_{\text {beat }}\right.$ in Eq. (1)] using two photodiodes in a resonant circuit. The resonant circuit enhances the signal-to-noise ratio at the resonant frequency of $65 \mathrm{MHz}$. The heterodyne detection scheme was designed for each detector to measure $f_{\text {beat }}$ $180^{\circ}$ out of phase, such that a subtraction of the two signals adds the desired signal while suppressing amplitude fluctuations that are common to both arms. The combination of this balanced detection and resonant enhancement in the detectors increased the signalto-noise ratio by approximately $5 \mathrm{~dB}$ to more than $25 \mathrm{~dB}$, which was critical for accurate counting. The beat signal was then filtered, amplified, and sent to a third frequency counter (counter 3 in Fig. 1). We determine the frequency of the He-Ne laser by adding the recorded RF frequencies via Eq. (1) after subtracting the frequency shift of the single side-band mixer.

For a valid comparison of our measurement to the International Committee for Weights and Measures
(CIPM) recommended value [18], the proper operating parameters must be used. All conditions are within the range of recommended values except for the intracavity optical power of our He-Ne laser. The CIPM recommends specific operating conditions for the iodine-stabilized He-Ne; recommended is a modulation depth of $6 \pm 0.3 \mathrm{MHz}$, a cold finger temperature of $15 \pm 0.2^{\circ} \mathrm{C}$, and a cell wall temperature of $25 \pm 5^{\circ} \mathrm{C}$, and an intracavity optical power of $10 \pm 5 \mathrm{~mW}$. Our commercial He-Ne laser operates with $6.0 \pm 0.3 \mathrm{MHz}$ modulation depth, a cold-finger temperature of $15.04{ }^{\circ} \mathrm{C}$, a cell wall temperature of $28 \pm 2{ }^{\circ} \mathrm{C}$, and an output power of $128 \mu \mathrm{W}$. Using the manufacturer estimated output coupler transmission of $0.7 \%$, our intracavity optical power, calculated to be $18.3 \mathrm{~mW}$, is nearly twice the CIPM recommended value of $10 \mathrm{~mW}$. We require the higher power to ensure sufficient signalto-noise of the beat between the He-Ne and a comb line. Though this intracavity power is outside the recommended range, we can correct for the frequency shift in the He-Ne laser that this causes based on empirical data from the manufacturer.

We test for the possibility of systematic errors in the detection of the He-Ne/comb beat ( $f_{\text {beat }}$ ) by altering the repetition rate to shift the comb line of interest to the opposite side of the He-Ne laser line and making another measurement. Because this reverses the sign of the $\mathrm{He}-\mathrm{Ne} / \mathrm{comb}$ beat in Eq. (1), systematic errors in the measurement of this beat would be evident in the resultant optical frequency measurements. This technique establishes an upper limit on the systematic error of $1.84 \mathrm{kHz}$ for each data set for the measurement of the $\mathrm{He}-\mathrm{Ne} / \mathrm{comb}$ beat.

\section{Results and Analysis}

We first analyze the stability of each of the three measured RF frequencies independently. The repetition rate $\left(f_{\text {rep }}\right)$, the carrier-envelope offset frequency $\left(f_{0}\right)$, and the He-Ne/comb beat frequency ( $\left.f_{\text {beat }}\right)$ exhibited standard deviations for a data set of $\sim 1000$ points (using a 1 second gate time) of $1.7 \mathrm{mHz}\left(\Delta f_{\text {rep }} / f_{\text {rep }}=\right.$ $\left.1.2 \times 10^{-11}\right), 4.8 \mathrm{mHz}\left(\Delta f_{0} / f_{0}=1.1 \times 10^{-8}\right)$, and $15.1 \mathrm{kHz}$ $\left(\Delta f_{\text {beat }} / f_{\text {beat }}=2.3 \times 10^{-4}\right)$, respectively (see Fig. 2(a-c)). The stabilities of and correlations between the three frequencies measured yield the fluctuations in the measured He-Ne frequency shown in Fig. 2(d). In order to lower the uncertainty in our measurement of the He-Ne frequency, we averaged the results from several measurements for a total of 2600 data points taken over the course of one day. 


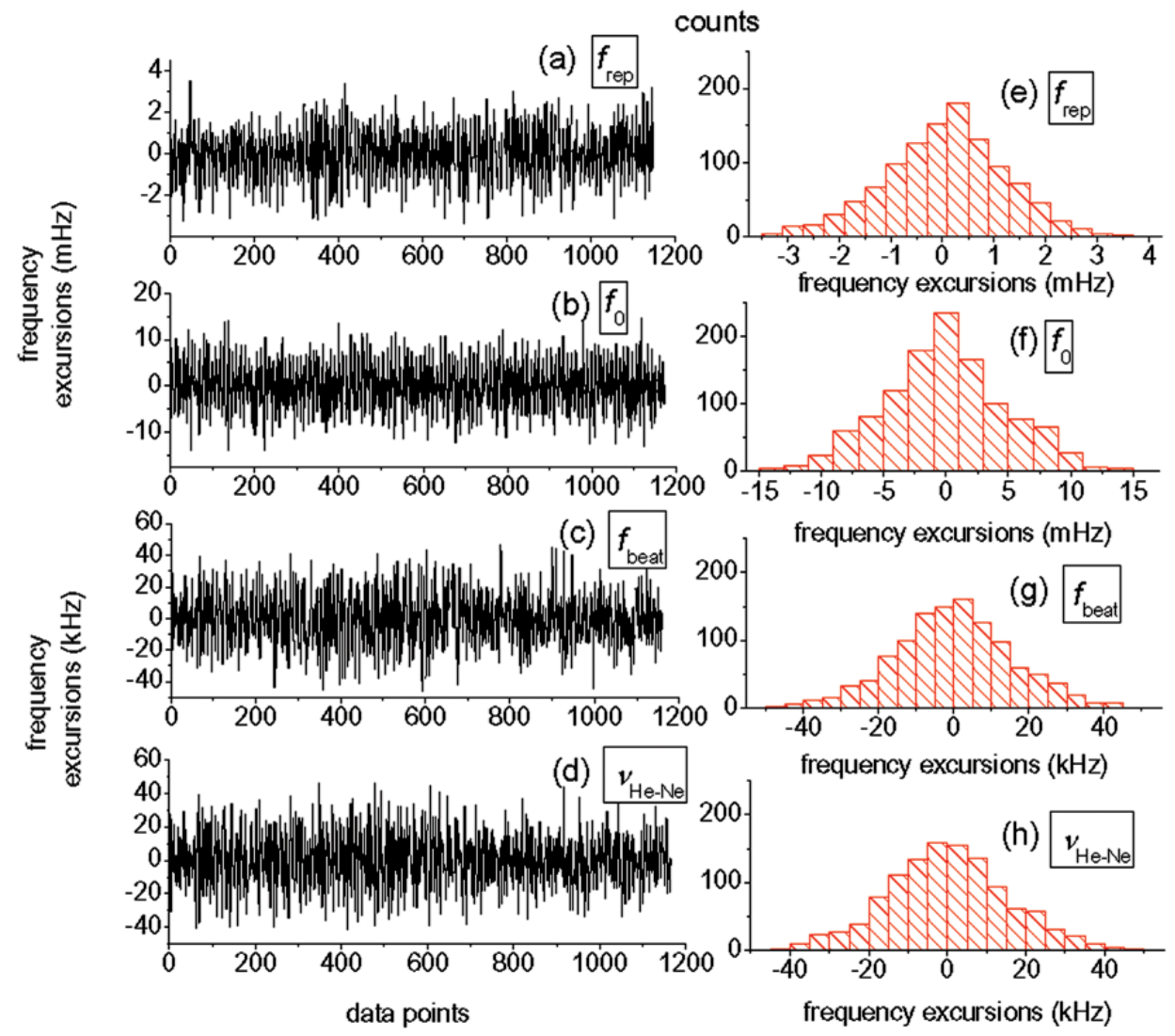

Fig. 2. Data from the longest continuous run. Outliers caused by cycle slips in one of the phase-lock loops are removed. Plots a-d show the frequency stability for the repetition rate, the carrier-envelope offset frequency, the beat frequency, and the calculated He-Ne laser frequency, respectively. Plots e-h are histograms for these frequencies.

We estimate the magnitudes of the sources of error in the measurement of the He-Ne frequency to ensure that we have properly considered relevant contributions and to determine the relative contribution to the error due to the new technique of measuring $f_{0}$. The fluctuations in $v_{\mathrm{He}-\mathrm{Ne}}$ can be found using an error propagation analysis [19] and Eq. (1) to be

$$
\begin{aligned}
& \sigma_{v_{\text {He-Ne }}}^{2}=n^{2} \sigma_{f_{\text {rep }}}^{2}+\sigma_{f_{\text {beat }}}^{2}+\sigma_{f_{0}}^{2} \pm 2 r_{f_{\text {rep f feat }}} n \sigma_{f_{\text {frep }}} \sigma_{\text {freat }} \\
& \quad \mp 2 r_{f_{0} f_{\text {beat }}} \sigma_{f_{0}} \sigma_{f_{\text {beat }}}-2 r_{f_{0} f_{\text {rep }}} n \sigma_{f_{0}} \sigma_{f_{\text {rep }}},
\end{aligned}
$$

where $\sigma_{x}^{2}$ is the variance of quantity $x, n$ is the known comb line index $\left(\sim 5 \times 10^{6}\right)$, and each $r_{x y}$ is a correlation coefficient calculated between quantity $x$ and quantity $y$. The contributions to the variance for the single longest run are given in Table 1. It is interesting to note that the uncertainty in $f_{\text {rep }}$ is almost exactly cancelled by the correlation term between $f_{\text {rep }}$ and $f_{\text {beat }}$. We attribute this to variations in the Cs reference frequency. Any fluctuations in the repetition rate due to instabilities of the Cs reference are not recorded in the composite measurement of the He-Ne laser frequency because the synthesizers and the counters share the same time-base.

Table 1. Contributions to the variance from each term in Eq. (2) for the single longest run ( 1100 data points). All values are in $\mathrm{Hz}^{2}$

\begin{tabular}{cccccc}
\hline \hline$n^{2} \sigma_{f_{\text {rep }}}^{2}$ & $\sigma_{f_{0}}^{2}$ & $\sigma_{f_{\text {beat }}}^{2}$ & $2 r_{f_{\text {rep }} f_{\text {beat }}} n \sigma_{f_{\text {rep }}} \sigma_{f_{\text {beat }}}$ & $2 r_{f_{0} f_{\text {beat }}} \sigma_{f_{0}} \sigma_{f_{\text {beat }}}$ & $2 r_{f_{0} f_{\text {rep }}} n \sigma_{f_{0}} \sigma_{f_{\text {rep }}}$ \\
\hline $3.5 \times 10^{7}$ & $2.3 \times 10^{-5}$ & $2.3 \times 10^{8}$ & $-3.4 \times 10^{7}$ & $1.1 \times 10^{0}$ & $1.6 \times 10^{1}$ \\
\hline
\end{tabular}


When the terms are appropriately added together using Eq. (2) and taking into account the correct sign for the He-Ne beat, the sum equals the variance of the measured $v_{\mathrm{He}-\mathrm{Ne}}$ within $0.2 \%$. It is clear from the table that the contribution to the variance due to the measurement of $f_{0}$ does not significantly reduce the precision of the measurement.

We perform Allan deviation and correlation calculations on our data to understand the stability of our measurement for different averaging times. We account for a dead time of $0.65 \mathrm{~s}$ between one second measurements. The Allan deviation analysis of the measured He-Ne frequency reveals a trend with averaging time of $\tau^{-1 / 2}$, a signature of a white frequency-modulation noise spectrum. This is very similar in trend to the cesium reference time-base noise. For this type of noise, trivial corrections for dead time are necessary, as opposed to the use of more complex bias functions [20]. Fig. 3 gives the Allan deviation of the measured He-Ne frequency, as well as independently recorded
Allan deviations for the He-Ne laser [15] and the cesium reference [21].

We correct for systematic shifts in the measured frequency because these are the only two conditions that necessitate a significant correction to our measured data. The output frequency depends on the pressure, which is related to the cold finger temperature in thermal equilibrium according to an empirical formula provided by the manufacturer. Pressure causes a collisional frequency shift of the iodine transition. We calculate that the correction due to the difference in temperature of the cell wall is $-0.493 \mathrm{kHz}$. The systematic shift related to optical power is due to the AC Stark effect, which causes the hyperfine levels of the atoms to repel. The correction of $+7.25 \mathrm{kHz}$ is also added to correct for an intracavity power of $18.3 \mathrm{~mW}$ (compared with the CIPM recommended value of $10 \mathrm{~mW}$ ). These two corrections were calculated from Taylor expansion coefficients provided by the manufacturer [22].

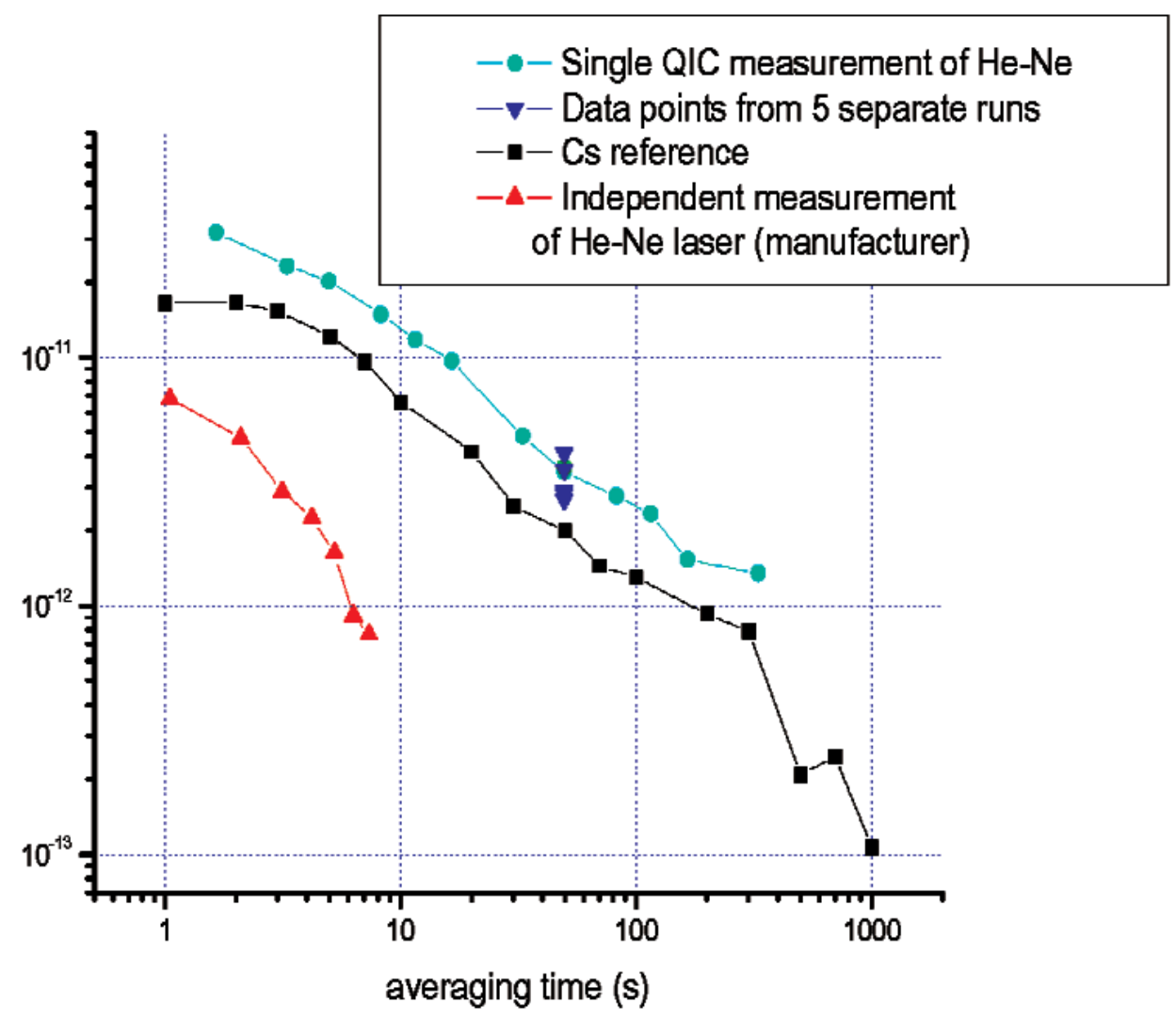

Fig. 3. Allan deviation of the measured He-Ne laser frequency for a single measurement using QIC, a cesium primary standard (data taken April 2004 at JILA), and an independent measurement of the Allan deviation for the He-Ne laser frequency being measured [18]. The other points shown at $50 \mathrm{~s}$ averaging time represent the Allan deviation computed for 5 shorter runs taken the same day as the single QIC measurement. 
Of the several data sets taken over the course of the day, the mean was $473612214712.96 \pm 0.663 \mathrm{kHz}$, which includes the two correctional shifts mentioned above. This value falls within the CIPM recommended range $473612214712 \pm 5$ kHz. Fig. 4 gives a comparison of the results of our measurements using both the standard $v$ - to $-2 v$ technique and QIC for the locking of the carrier-envelope offset frequency with the CIPM standard. Although no improvement in the error can be conclusively attributed to the QIC method, the error may potentially be reduced if a device could be engineered to generate an octave spanning spectrum in the same semiconductor as the carrier-envelope offset frequency is detected, thus reducing the number of components in the setup [23].

\section{Conclusion}

We have used a femtosecond comb stabilized via quantum interference control to make an accurate and precise measurement of the frequency of an iodinestabilized He-Ne laser. For averaging times of $330 \mathrm{~s}$, the Allan deviation was $663 \mathrm{~Hz}$. The frequency of the laser measured, after systematic correction shifts, was well within the CIPM recommended value for the ${ }^{127} \mathrm{I}_{2}$ $\mathrm{R}(127)$ 11-5 i component. The fluctuations in the QIC measurement compare well with the fluctuations in the same measurement using the $v$ - to $-2 v$ technique.

\section{References}

[1] F. Riehle, Frequency Standards: Basics and Applications, Wiley-VCH Verlag GmbH \& Co. KGaA, Weinheim, 2004.

[2] L. Hollberg, C. W. Oates, G. Wilpers, C. W. Hoyt, Z. W. Barber, S. A. Diddams, W. H. Oskay, and J. C. Bergquist, Optical frequency/wavelength references, J. Phys. B 38, S469-S495 (2005).

[3] D. J. Jones, S. A. Diddams, J. K. Ranka, A. Stentz, R. S. Windeler, J. L. Hall, and S. T. Cundiff, Carrier-Envelope Phase Control of Femtosecond Mode-Locked Lasers and Direct Optical Frequency Synthesis, Science 288, 635 (2000).

[4] S. A. Diddams, D. J. Jones, J. Ye, S. T. Cundiff, J. L. Hall, J. K. Ranka, R. S. Windeler, R. Holzwarth, T. Udem, and T. W. Hänsch, Direct Link between Microwave and Optical Frequencies with a $300 \mathrm{THz}$ Femtosecond Laser Comb, Phys. Rev. Lett. 84, 5102 (2000).

[5] Th. Udem, R. Holzwarth, and T. W. Hänsch, Optical frequency metrology, Nature 416, 233-237 (2002).

[6] S. T. Cundiff and J. Ye, Colloquium: Femtosecond optical frequency combs, Rev. Mod. Phys. 75, 325 (2003).

[7] M. M. Boyd, A. D. Ludlow, S. Blatt, S. M. Foreman, T. Ido, T. Zelevinsky, and J. Ye, ${ }^{87} \mathrm{Sr}$ Lattice Clock with Inaccuracy below $10^{-15}$, Phys. Rev. Lett. 98, 083002 (2007).

[8] W. H. Oskay, S. A. Diddams, E. A. Donley, T. M. Fortier, T. P. Heavner, L. Hollberg, W. M. Itano, S. R. Jefferts, M. J. Delaney, K. Kim, F. Levi, T. E. Parker, and J. C. Bergquist, Single-Atom Optical Clock with High Accuracy, Phys. Rev. Lett. 97, 020801 (2006).

[9] S. T. Cundiff, J. Ye, and J. L. Hall, Optical frequency synthesis based on mode-locked lasers, Rev. Sci. Instr. 72, 3749 (2001).

[10] A. Haché, J. E. Sipe, and H. M. van Driel, Quantum Interference Control of Electrical Currents in GaAs, IEEE J. Quantum Electron. 34, 1144-1154 (1998).

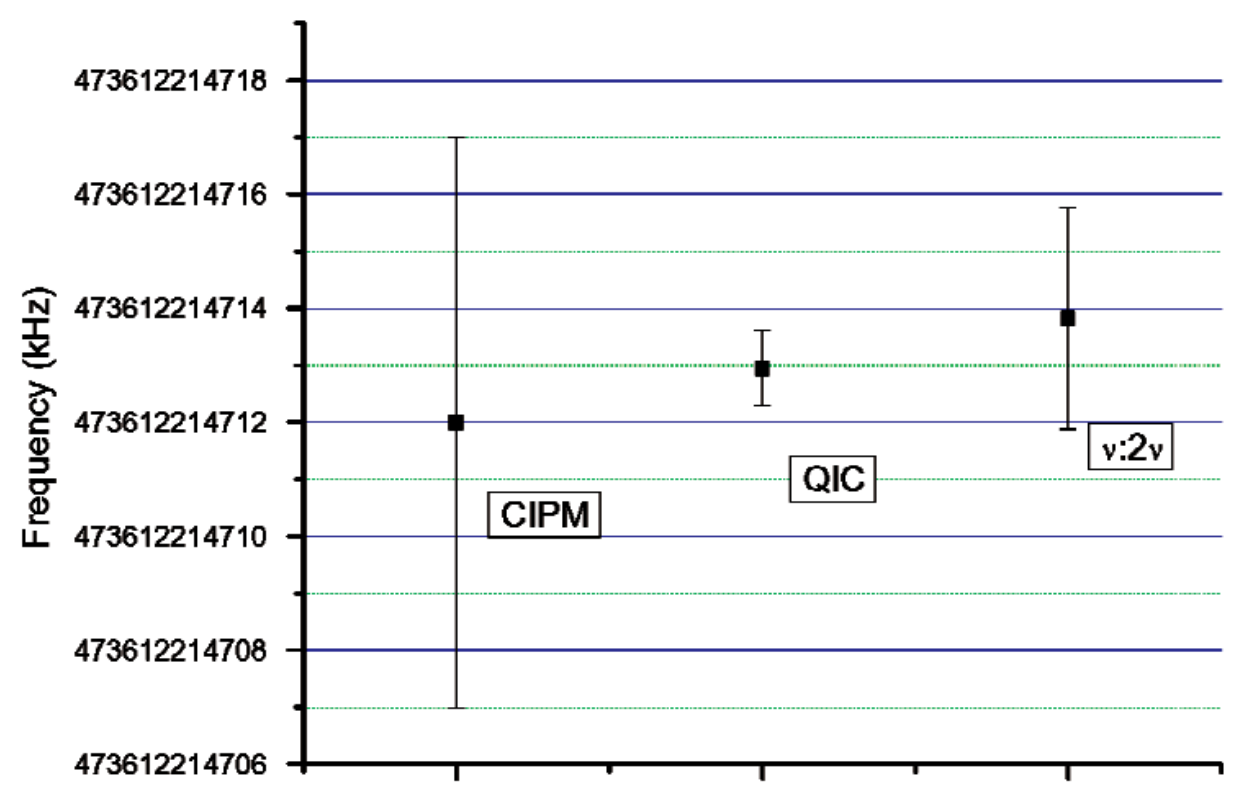

Fig. 4. Comparison between QIC and $v$ - to $-2 v$ the standard self-referencing technique for optical comb metrology. Both results fall within the uncertainty of the CIPM recommended values. Allan deviations at the best averaging time in our measurements are $0.663 \mathrm{kHz}$ for QIC, and $1.944 \mathrm{kHz}$ for $v$ - to $-2 v$. 
[11] H. M. van Driel and J. E. Sipe, in Ultrafast Phenomena in Semiconductors, (Ed. K. T. Tsen, Springer-Verlag, New York 2001) p. 261, and references therein.

[12] P. Roos, Q. Quraishi, S. T. Cundiff, R. Bhat, and J. Sipe, Characterization of quantum interference control of injected currents in LT-GaAs for carrier-envelope phase measurements, Opt. Express 11, 2081-2090 (2003).

[13] T. M. Fortier, P. A. Roos, D. J. Jones, S. T. Cundiff, R. D. R. Bhat, and J. E. Sipe, Carrier-Envelope Phase Controlled Quantum Interference of Injected Photocurrents in Semiconductors, Phys. Rev. Lett. 92, 147403 (2004).

[14] P. A. Roos, X. Li, R. P. Smith, J. A. Pipis, T. M. Fortier, and S. T. Cundiff, Solid-state carrier-envelope phase stabilization via quantum interference control of injected photocurrents, Opt. Lett. 30, 735-737 (2005).

[15] Mike Winters, Operator's Manual: Model 100 IodineStabilized HeNe Laser, Winters Electro-Optics, Inc., Colorado (1994).

[16] P. A. Roos, X. Li, J. A. Pipis, T. M. Fortier, and S. T. Cundiff, Characterization of carrier-envelope phase-sensitive photocurrent injection in a semiconductor, J. Opt. Soc. Am. B 22, 362-368 (2005).

[17] J. Reichert, R. Holzwarth, T. Udem, and T. W. Haensch, Measuring the frequency of light with mode-locked lasers, Opt. Commun. 172, 59 (1999).

[18] T. J. Quinn, Practical realization of the definition of the metre, including recommended radiations of other optical frequency standards, Metrologia 40, 103-133 (2003).

[19] J. R. Taylor, An Introduction to Error Analysis: The Study of Uncertainties in Physical Measurements, University Science Books, California (1982).

[20] J. Barnes and D. Allan, Variances on data with dead time between the measurements, NIST Technical Note 1318, 1990.

[21] Kevin W. Holman, Distribution of an Ultrastable Frequency Reference Using Optical Frequency Combs, JILA thesis, 2005.

[22] Private correspondence with Mike Winters, manufacturer of the Winters Electro-Optics Model 100.

[23] O. D. Mücke, T. Tritschler, M. Wegener, U. Morgner, and F. X. Kärtner, Determining the carrier-envelope offset frequency of 5 -fs pulses with extreme nonlinear optics in $\mathrm{ZnO}$, Opt. Lett. 27, 2127-2129 (2002).

About the authors: Ryan P. Smith is a graduate student in the physics department at the University of Colorado currently working at JILA. Peter A. Roos contributed to this work as a post-doc at JILA, and is presently a research scientist at the Montana State University Spectrum Lab. Jared K. Wahlstrand is a post-doc at JILA. Jessica A. Pipis contributed to this work as a professional research assistant at JILA. Maria Belmonte Rivas contributed to this work as a graduate student in the aerospace engineering department at the University of Colorado. Steven T. Cundiff is a member of the NIST Quantum Physics Division, a JILA Fellow and an Associate Professor Adjoint with the Departments of Physics and Electrical and Computer Engineering at the University of Colorado. The National Institute of Standards and Technology is an agency of the U.S. Department of Commerce. 